\title{
15 PFO CLOSURE REDUCES PLASMA LEVELS OF SEROTONIN IN A LONG TERM FOLLOWUP OF STROKE PATIENTS
}

W Deng, D McMullin, T Wickham, K Feeney, I Inglessis, I Palacios, FS Buonanno, EH Lo, M Ning. Neurology, Massachusetts General Hospital, Boston, MA, United States

\subsection{6/jim-2016-000080.31}

Purpose of Study PFO allows venous clots and vasoactive factors to bypass pulmonary filtration and remain in circulation. We previously identified an immediate reduction of procoagulant serotonin (5-HT) in left atrial blood post PFO closure. To understand the long-term effect of PFO closure, we report the change of 5-HT in peripheral venous blood in 1-year followup.

Methods Used 97 PFO-related stroke patients were recruited on IRB approval. Venous blood was collected at baseline (BL) and 1 year follow-up (FU) of treatments (PFO closure and medical therapy). Plasma 5-HT was quantified by mass spectrometry. Patients with serotonin modifying medications (ie. SSRIs) or conditions (anxiety/ depression) were excluded.

Summary of Results 5-HT level in peripheral venous blood was significantly reduced by $27.27 \%$ (BL: 7.57
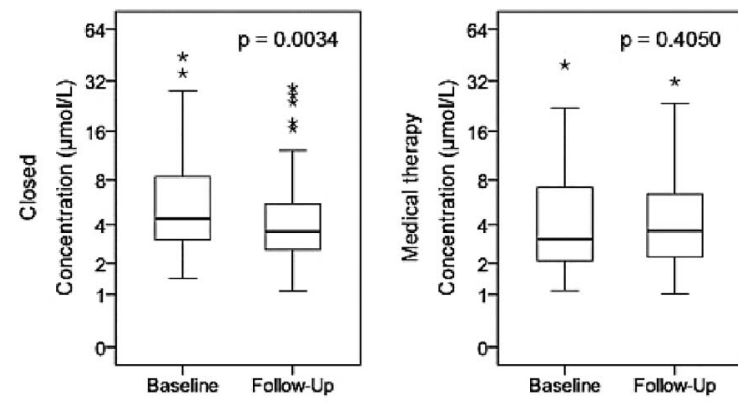

\begin{tabular}{cccc}
\hline Linear regression & $\mathrm{B}$ & $\mathbf{9 5 \%} \mathrm{Cl}$ & P-value \\
\hline treatment & 0.523 & $(0.001,1.045)$ & 0.0497 \\
Age & -0.017 & $(-0.036,0.003)$ & 0.0909 \\
Gender & -0.382 & $(-0.901,0.137)$ & 0.147 \\
Medical history & & & \\
Hyperlipidemia & -0.194 & $(-0.777,0.39)$ & 0.5112 \\
Hypertension & -0.457 & $(-1.034,0.12)$ & 0.1193 \\
$\quad$ Dlabetes & -0.203 & $(-1.357,0.952)$ & 0.7281 \\
TIA/Stroke/Migraine & 0.192 & $(-0.399,0.784)$ & 0.5201 \\
Medication & & & \\
$\quad$ Antiplatelet & -0.283 & $(-0.988,0.421)$ & 0.4267 \\
Anticoagulation & 0.515 & $(-0.319,1.348)$ & 0.2233 \\
Lipid-lowing Agent & -0.268 & $(-0.782,0.245)$ & 0.3019 \\
Antlhypertenslon Agent & 0.114 & $(-0.444,0.673)$ & 0.6856 \\
\hline
\end{tabular}

Abstract 15 Figure 1 
$\pm 8.04 \mu \mathrm{M}$; FU: $5.51 \pm 5.72 \mu \mathrm{M} ; \mathrm{p}=0.0034)$ in 61 patients receiving PFO closure (figure $1 \mathrm{~A})$. In the $37 \mathrm{PFO}$ patients treated with medicine alone, no changes were observed (BL: $5.79 \pm 7.15 \mu \mathrm{M}$; FU: $6.25 \pm 6.68 \mu \mathrm{M} ; \mathrm{p}=0.4050$ ) (figure 1B). 5-HT reduction was independently associated with PFO closure after adjusting for age, gender, medical history and medication status in a multivariate regression (figure 1C).

Conclusions We found that PFO closure independently reduced 5-HT level in peripheral venous blood. These results support the hypothesis that PFO related right-to-left interatrial shunt may foster higher level of procoagulant and vascoactive substances in circulation. While PFO closure decrease prothrombotic markers immediately post closure, this effect is sustained in long term followup up to 1 year. Further studies on the clinical outcome of these PFO patients with respect to their prothrombotic circulatory profiles are ongoing. 